\title{
Les collections d'instruments de musique au futur musée du quai Branly
}

\section{Madeleine Leclair}

\section{(2) OpenEdition}

Journals

Édition électronique

URL : http://journals.openedition.org/ethnomusicologie/595

ISSN : 2235-7688

Éditeur

ADEM - Ateliers d'ethnomusicologie

Édition imprimée

Date de publication : 1 novembre 2003

Pagination : 179-188

ISBN : 978-2-8257-0863-7

ISSN : 1662-372X

\section{Référence électronique}

Madeleine Leclair, «Les collections d'instruments de musique au futur musée du quai Branly », Cahiers d'ethnomusicologie [En ligne], 16 | 2003, mis en ligne le 16 janvier 2012, consulté le 19 avril 2019. URL: http://journals.openedition.org/ethnomusicologie/595

Ce document a été généré automatiquement le 19 avril 2019

Tous droits réservés 


\title{
Les collections d'instruments de musique au futur musée du quai Branly
}

\author{
Madeleine Leclair
}

1 La collection d'instruments de musique qui sera conservée au futur musée du quai Branly est constituée d'environ huit mille sept cents instruments de musique de toutes provenances. Elle regroupe les collections qui se trouvaient autrefois au Musée National des Arts d'Afrique et d'Océanie (MNAAO) et au département d'ethnomusicologie du Musée de l'Homme (MH).

2 Les quelque quatre cents instruments de musique provenant des collections du MNAAO étaient répartis dans les quatre départements de ce musée: Afrique subsaharienne et Océanie, qui comprenaient le plus grand nombre d'instruments, Maghreb et Fonds historique. Dans ce dernier département étaient conservés certains objets témoins de l'histoire du musée, inauguré à la suite de l'Exposition Coloniale en 1931. Avant qu'ils ne soient réunis et qu'ils n'intègrent les réserves de ce qui sera le futur département d'ethnomusicologie du musée du quai Branly, ces instruments ne constituaient pas une collection à part entière. Leur histoire est donc intimement liée à celle de chacun des départements qui avaient la responsabilité de leur gestion, de leur conservation et de leur mise en valeur.

3 De la collection comprenant huit mille trois cents instruments de musique naguère conservée au département d'ethnomusicologie du Musée de l'Homme, on connaît la richesse, la grande diversité et le prestige de celui grâce à qui elle fut constituée : André Schaeffner. On apprécie les importants travaux de recherche qui ont porté sur cette collection, aussi bien dans le domaine de l'organologie classificatoire qu'en ce qui concerne l'organologie régionale. L'ouvrage majeur d'André Schaeffner Origine des instruments de musique auquel il a travaillé de 1930 à 1936 est un classique de l'ethnomusicologie, tout comme l'est le livre de Curt Sachs Les instruments de musique de Madagascar publié en 1938, fondé sur l'étude des instruments de musique du Musée de l'Homme. On connaît également le travail remarquable mené par Geneviève Dournon 
pendant près de vingt-cinq ans, lorsqu'elle était chargée de ces collections instrumentales. Outre le Salon de musique qu'elle créa en 1985, salle d'exposition permanente des instruments qui avait pour but de "montrer à un large public d'une manière claire et attrayante, l'importance et la diversité du phénomène musical à travers quelques-uns de ses aspects et dans une perspective universelle» (1993: 3), elle a conservé, étudié et décrit les collections, et publié d'importants travaux touchant à l'organologie musicale ainsi qu'au travail muséographique. Son Guide pour la collecte des musiques et instruments traditionnels (1996), publié en trois langues, est un outil indispensable pour l'enquête ethnomusicologique sur le terrain. Ce travail de gestion et de mise en valeur des collections instrumentales fut poursuivi par Lucie Rault qui, depuis 1995, contribue à les faire connaître à un large public par le biais de nombreuses expositions et publications, notamment le très bel ouvrage Instruments de musique du Monde (2000).

4 Entre l'année 1878 où ont été réunis au Musée d'Ethnographie du Trocadéro (MET) environ cent cinquante instruments de musique dont la plupart étaient des pièces archéologiques, et l'année 2000 où les instruments réunis au Musée de l'Homme en sont venus à constituer l'une des plus riches collections comparatives d'instruments de musique en Europe, quels ont été les événements déterminants pour l'accroissement et la constitution de cet ensemble? C'est cette facette importante du passé muséographique de la collection que j'aimerais évoquer brièvement dans ces quelques pages.

5 L'une des premières tâches que j'ai accomplies en vue d'organiser les réserves instrumentales du futur musée du quai Branly a été de procéder à l'inventaire complet et informatisé de ces instruments. De cet inventaire, j'ai pu établir un schéma qui rend compte de l'accroissement progressif des collections instrumentales de musique provenant d'Afrique, d'Amérique, d'Asie, d'Europe et d'Océanie entre les années 1878 et $2000^{1}$.

6 Ces courbes qui se croisent ou au contraire s'éloignent au fil du temps permettent d'envisager quatre périodes importantes dans l'histoire de la constitution de cette collection. La première va de 1878 à environ 1910 ; elle est suivie d'une seconde période qui va de 1910 à 1928 au cours de laquelle très peu d'instruments de musique sont entrés au musée. Un accroissement spectaculaire des collections instrumentales a lieu entre les années 1928 et 1940. Après la fin de la guerre en 1945, les collections continueront de s'enrichir progressivement. Mais l'accent sera davantage mis sur la collecte et la publication des musiques enregistrées par les chercheurs: constitution d'archives sonores, réalisation de films, collections des chercheurs du Musée de l'Homme.

$7 \mathrm{Si}$ ces données d'ordre purement quantitatif soulèvent un certain nombre d'interrogations concernant le développement de la recherche ethnomusicologique dans les grandes institutions parisiennes ou encore l'enseignement de cette discipline, elles incitent également à s'interroger sur l'histoire de la constitution des collections ethnographiques, et plus spécifiquement ethnomusicologiques, au sein d'institutions muséales. 
Schéma représentant l'accroissement des collections instrumentales conservées au Musée de l'Homme

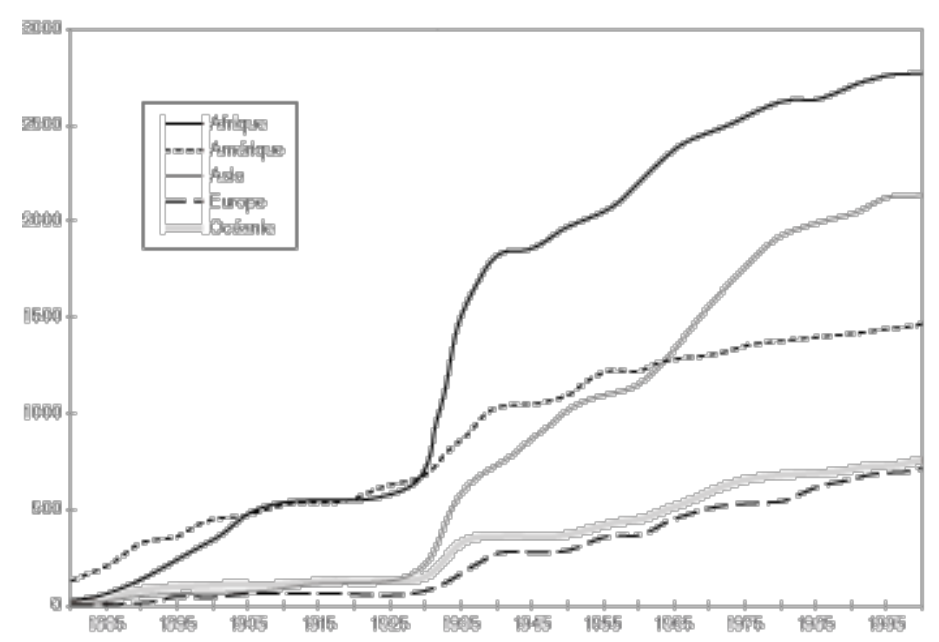

L'axe vertical correspond au nombre d'instruments de musique tandis que l'axe horizontal est celui du temps.

8 L'histoire du Musée d'Ethnographie du Trocadéro (MET), fondé en 1878, et de sa transformation en ce qui deviendra le Musée de l'Homme à partir de 1937 est relativement bien connue, grâce notamment à de nombreux articles publiés par Jean Jamin, à l'ouvrage de Nélia Dias (1991) et à l'étude réalisée par Fabrice Grognet (1997-98). Les moments clés de l'histoire de ces deux institutions sont clairement exposés dans ces deux travaux de recherche. C'est à la lumière de ces données que je me propose de passer rapidement en revue quelques faits importants concernant les instruments de musique qui y étaient conservés.

\section{La constitution des collections au Musée d'Ethnographie du Trocadéro et au Musée de l'Homme 2}

9 L'idée de rassembler à Paris, en un même endroit, des objets ethnographiques disséminés dans divers lieux apparaît dès 1831. Cette initiative est due à Edmé-François Jomard, nommé par Charles $\mathrm{X}$ ingénieur et conservateur du dépôt de Géographie à la Bibliothèque du Roi : «L'état actuel des sciences géographiques appelle à la formation d'une collection spéciale destinée à recevoir les produits des voyages lointains, et qui sont propres à éclaircir les mœurs et les usages des nations et des peuplades non connues. Tel serait l'objet principal d'une collection ethnographique, supplément utile ou même nécessaire aux descriptions géographiques et aux relations des découvertes » (E.-F. Jomard 1831, cité in Grognet 1997-98: 10).

Ce projet, longtemps resté sans suite, put finalement être réalisé en 1878 grâce à l'Exposition Universelle qui eut lieu à Paris cette même année, et à l'occasion de laquelle fut construit le Musée d'Ethnographie du Trocadéro.

Durant la période qui va de 1878 à 1928, l'étude des objets consistait avant tout en une démarche de travail en laboratoire conduite par des anthropologues et conservateurs chargés de la gestion et de la conception muséographique, qui n'avaient pas eux-mêmes 
participé à la constitution des collections, puisqu'ils ne participaient pas à la collecte d'objets sur le terrain. Ainsi, les instruments de musique acquis par le MET à cette époque sont bien souvent accompagnés de fiches descriptives lacunaires en ce qui concerne leur origine exacte ou leur fonction. Cette réalité est d'autant plus forte que l'attribution d'un numéro d'inventaire à chaque instrument, seul et unique moyen de rattacher un objet à sa collection d'origine, ne s'est pas faite de manière systématique, faute de ressources financières et très certainement aussi par manque de personnel travaillant au MET durant ces années.

En effet, de 1878 à 1928, le MET souffre d'un important manque de financement qui serait dû au désintérêt de l'État à l'égard de cette institution qu'il avait pourtant lui-même créée. Cette lente dégradation de l'institution, qui était pourtant l'une des vitrines importantes de la France lors de l'Exposition Universelle, se traduit bien entendu directement par un appauvrissement des collections ethnographiques. Les collections instrumentales continuent de s'enrichir jusque dans les années 1910, mais cette situation est essentiellement due à des dons de voyageurs ou collectionneurs particuliers ainsi qu'à des dépôts d'institutions gouvernementales, muséographiques ou non. En raison du peu de moyens que le MET pouvait investir dans le travail de recherche scientifique mené sur le terrain, et du fait que la recherche ethnographique dans ce musée n'était pas encore institutionnalisée à cette époque, peu d'instruments provenant de missions de recherche ont été acquis. Si l'on en juge par la très faible croissance des collections instrumentales de 1910 à 1928 - environ deux cent cinquante instruments de musique sont entrés au MET pendant ces dix-huit années - on peut penser qu'à cette époque, les difficultés institutionnelles de ce musée étaient loin de s'être améliorées.

De 1928 à 1940, la conjugaison de plusieurs événements liés au développement de l'ethnographie et à l'effervescence artistique de l'époque marque un tournant décisif dans l'histoire de la constitution des collections ethnographiques du MET. Mon intention n'est pas ici de chercher à rendre compte du foisonnement intellectuel et culturel qui a marqué ces années d'avant-guerre, mais de rappeler brièvement certains faits relatifs à cette époque, qui ont sans doute présidé à la nouvelle orientation qu'a pris à partir de ce moment le travail de collecte et d'étude des collections instrumentales.

Paul Rivet, anthropologue américaniste, est nommé directeur du MET en 1928. GeorgesHenri Rivière le rejoint en 1929. Ensemble, ils réorganisent le Musée d'Ethnographie du Trocadéro, qui deviendra le Musée de l'Homme en 1937. Sous la direction de Paul Rivet, Georges-Henri Rivière présentera quelque 70 expositions de 1928 à 1937, année où il quitte le Musée de l'Homme pour travailler à un autre projet muséal : le Musée des Arts et Traditions Populaires.

15 Aussitôt nommé à la tête du MET, Paul Rivet procéda au rattachement officiel de ce musée à la chaire d'anthropologie du Muséum National d'Histoire Naturelle, qui se transforma alors en une "Chaire d'Ethnologie des hommes actuels et des hommes fossiles ». Sitôt rattaché à la communauté scientifique, le musée se vit allouer des crédits substantiels grâce auxquels de très importantes missions de recherche et de collecte sur le terrain purent être organisées, comme par exemple la célèbre mission Dakar-Djibouti (mai 1931février 1933) conduite par Marcel Griaule et Michel Leiris, et à laquelle a notamment participé André Schaeffner.

$16 \mathrm{Au}$ cours de cette mission, trois mille six cents objets dont environ deux cents instruments de musique auront été collectés sur le terrain, selon un protocole qui avait été établi à cette intention et publié par le MET sous le titre de Instructions sommaires 
pour les collecteurs d'objets ethnographiques. Ce manuel de collecte sur le terrain à l'usage des ethnologues mais aussi des « fonctionnaires, voyageurs, touristes, colons [...] en rapports directs et journaliers avec les habitants des régions qu'ils traversent ou dans lesquelles ils vivent » (Grognet 1997-98: 50) encourage les chercheurs à procéder à des collectes systématiques d'objets mais aussi à recueillir un maximum de données ethnographiques. C'est notamment en raison de cette initiative que les fiches descriptives relatives aux instruments de musique qui ont été collectés à partir de ces années, sur lesquelles est souvent consigné un ensemble riche et précis de données ethnographiques, ont pu être rédigées.

17 Alors qu'auparavant la collecte et la recherche étaient réalisées par des personnalités qui œuvraient chacune de leur côté, à partir des années ' 30 ces deux fonctions sont assumées par un même groupe de personnes qui alliaient la théorie à la pratique, démarche propre à la recherche en ethnologie et en ethnomusicologie. Du fait que sur le terrain s'opérait une sélection selon des critères scientifiques, les instruments de musique qui entreront désormais au musée formeront, pour une très grande partie, de véritables ensembles culturellement cohérents.

C'est cet esprit de rigueur et de cohérence scientifique qui animera les chercheurs ethnomusicologues lorsqu'ils procéderont par la suite à la collecte d'instruments de musique sur le terrain. Le Département d'ethnologie musicale du MET, aujourd'hui département d'ethnomusicologie du Musée de l'Homme, est créé en 1929 par André Schaeffner avec le soutien de Georges-Henri Rivière et de Paul Rivet. Par la suite, ce département accueillera une phonothèque et un laboratoire d'analyse du son et deviendra un important centre de recherche et d'enseignement de l'ethnomusicologie ${ }^{3}$. Plusieurs chercheurs attachés à ce département contribueront à enrichir les collections d'instruments de musique conservés au Musée de l'Homme.

On remarque qu'au cours des années 1928-1940, c'est la collection d'instruments de musique d'Afrique qui connaîtra l'accroissement le plus spectaculaire. Les raisons de cette importante expansion sont multiples. Elles sont certainement en rapport avec l'investissement que l'État français développait pour les colonies africaines durant ces années, mais aussi avec l'engouement de la bourgeoisie parisienne pour le jazz, et l'exotisme que représentaient les musiques africaines à l'époque.

Si Georges-Henri Rivière, pianiste de jazz, compositeur et accompagnateur de Joséphine

Baker lors de sa venue à Paris dans le cadre de la Revue Nègre en $1925^{4}$, et André Schaeffner, à qui l'on doit le premier ouvrage en langue française sur le jazz qu'il a coécrit avec André Coeuroy et dans lequel il tente de tracer les racines africaines et afroaméricaines de cette musique (Coeuroy \& Schaeffner 1988), jouèrent un rôle décisif dans les années 1930 quant à la pénétration du jazz en France, ils jouèrent également un rôle incontournable dans le domaine de la muséographie durant ces années. C'est probablement en partie à leur initiative que les collections d'instruments de musique provenant d'Afrique ont connu un essor aussi important.

21 Par ailleurs, les coupures de presse conservées dans les archives du Musée de l'Homme concernant ces années sont tout à fait révélatrices de l'esprit particulièrement novateur de cette époque. Ces archives témoignent aussi de l'effervescence et de la quête de contemporanéité qui animait ceux qui travaillaient au musée, et permettent de constater la place prépondérante que la musique occupait dans la programmation des expositions présentées au public par le musée. 
22 Par exemple, l'exposition organisée par Henri Labouret au retour de son importante mission de collecte et de recherche au Cameroun, "La musique indigène au Cameroun », fut accompagnée d'une série de concerts. Comme pour les autres manifestations de ce genre à l'époque, il s'agissait en fait de ce qu'on appelait un "concert de disques ", autrement dit d'une écoute publique de musique enregistrée.

Dans son article «Une visite au Musée d'Ethnographie du Trocadéro où l'on trouve les plus riches collections du monde » (1935), René Thévenin rend compte de ce que sont les nouvelles salles d'exposition et conclut son texte en ces termes :

[...] il est une autre section dont je ne saurais trop vous signaler l'importance et l'intérêt: tous les samedis, de 16 à 17 heures, a lieu un concert de disques enregistrés sur place, reproduisant ces chants ou ces mélodies exotiques d'un charme si captivant, et que bientôt on ne pourra plus même entendre dans leurs pays d'origine. Pour peu que vous soyez sensibles à ce charme, vous trouverez, aux samedis du Trocadéro, de quoi vous procurer complète satisfaction [...] par l'audition de véritables thèmes indigènes, d'une originalité unique, qui vous sera la meilleure garantie de leur authenticité. Or, ceux de ces disques qui vous plaisent, vous pourrez les acquérir et les rejouer à votre tour sur votre phonographe. C'est là une innovation des plus intéressantes et dont tous les amateurs d'art doivent profiter.

Enfin, une autre annonce parue en décembre 1938 dans la Revue Internationale de Sociologie (Paris) donne une idée de l'importance des activités du musée liées à la musique : «Le Musée est ouvert de 21 heures à $23 \mathrm{~h} 30$ les mercredi, jeudi, vendredi et samedi. Tous les jours, l'après-midi de 15 à 17 heures, audition de musique exotique enregistrée. »

Durant la période qui va de l'après-guerre jusqu'en 2000, le département d'ethnomusicologie diversifie et multiplie ses activités de recherche et de diffusion, allant de pair avec un accroissement soutenu des collections de ce département, notamment pour celles qui proviennent des continents africain et asiatique.

26 Parmi les événements les plus marquants de ces années, mentionnons la première grande mission ethnomusicologique, la mission « Ogooué-Congo » en Afrique équatoriale, qui fut organisée en 1946. André Didier, professeur au Conservatoire national des arts et métiers et Gilbert Rouget, assisté de l'explorateur Pierre-Dominique Gaisseau, y enregistreront plus de six cents disques de gravure directe de musique bantou et pygmée.

27 Quelques deux cents instruments de musique du Cameroun et du Niger seront rapportés au musée par Marcel Griaule lors de ses deux importantes missions de recherche de 1938 et de 1959.

28 Cette même année est inaugurée au Musée de l'Homme la Salle des Arts et Technique dont une grande section consacrée aux instruments de musique fut créée par André Schaeffner. Entièrement repensée et réorganisée par Genevière Dournon, elle deviendra en 1985 le Salon de Musique.

29 L'enrichissement de la collection des instruments de musique d'Asie est, au cours de cette période, autant lié à d'importants dons et dépôts qui, entre 1943 et 1962, seront consentis par d'autres institutions muséographiques - Musée Guimet, Musée de la Marine et autres - qu'aux collectes faites par des ethnologues et ethnomusicologues lors de missions de recherche sur le terrain, comme celles que Corneille Jest a accomplies au Népal et au Bhoutan entre 1961 et 1985 et dont il rapporta plus de soixante-quinze instruments, ou celles menées par Genevière Dournon dans les régions du centre et du nord de l'Inde 
entre 1972 et 1992, qui lui ont permis de recueillir plus d'une centaine d'instruments de musique.

\section{Perspectives pour l'avenir}

Depuis des dizaines d'années, les conservateurs et muséographes ayant la responsabilité de collections instrumentales savent que la valeur d'un instrument de musique tient autant aux particularités de sa forme et de sa facture qu'aux propriétés de son fonctionnement acoustique. Aussi importante et diversifiée qu'elle soit, une collection d'instruments de musique ne peut pas à elle seule suffire à exposer dans un musée le domaine des musiques du monde.

Le projet muséographique que je souhaiterais voir mis en oeuvre dans les espaces d'exposition permanente du musée du quai Branly porte sur un ensemble de thématiques qui, reliées l'une à l'autre, forment une sorte de continuum dont les limites extrêmes concernent deux formes d'expression artistique essentiellement différentes bien qu'étant dépendantes l'une de l'autre : l'instrument de musique considéré pour la valeur plastique de sa construction, et la musique envisagée comme une forme d'art à part entière. Il existe, entre ces deux pôles, un grand nombre de sujets ou de thèmes qui pourraient faire l'objet d'un traitement muséographique. Parmi eux, ceux qui sont retenus ou à retenir en priorité dans un avenir proche visent le même objectif : proposer au public des clés de compréhension pour les différents aspects de la facture instrumentale et de l'exécution musicale.

2 Les collections instrumentales autrefois conservées au Musée de l'Homme et au MNAAO seront réunies dans la future réserve du musée du quai Branly destinée à la conservation des instruments de musique. Dans cette réserve, qui se présente sous la forme d'un grand cylindre de verre traversant les différents niveaux du bâtiment, les instruments de musique seront d'abord regroupés selon leur origine géographique puis selon des critères typologiques. Par des dispositifs d'éclairage qui s'adapteront aux contraintes de conservation des collections, à la structure architecturale et à l'environnement muséographique général, certaines parties de cette réserve seront partiellement visibles tandis que d'autres seront volontairement obscurcies.

En dehors du projet muséal envisagé pour chacune des quatre grandes zones d'exposition permanente du musée, qui correspondent chacune à une aire géographique et dans lesquelles plus d'une centaine d'instruments seront intégrés et musicalement illustrés, trois programmes muséographiques destinés à la mise en valeur des collections ethnomusicologiques seront répartis autour de cet axe transversal que représente la réserve des instruments de musique. Ces programmes, qui sont actuellement en cours d'élaboration, s'articulent autour des thématiques suivantes :

La facture instrumentale et les techniques de jeu des instruments pourraient donner lieu à des programmes consultables à partir d'un certain nombre de dispositifs placés autour de la réserve des instruments.

Dans deux espaces fermés d'environ $35 \mathrm{~m}^{2}$ situés en des lieux différents du musée sont prévues des installations multimédia qui visent mettre à la disposition du public des points de repère auditifs et visuels pour la découverte de répertoires musicaux et chorégraphiques appartenant aux cultures de tradition orale. 

l'anthropologie, est prévue la réalisation d'un projet consistant à proposer aux visiteurs d'explorer et d'approfondir un certain nombre de notions concernant la recherche ethnomusicologique.

\section{BIBLIOGRAPHIE}

COEUROY André et André SCHAEFFNER, 1988 [1926], Le jazz. Paris : Éditions Claude Aveline.

DIAS Nélia, 1991, Le Musée d'Ethnographie du Trocadéro : 1878-1908. Anthropologie et muséologie en France. Paris : éditions du CNRS.

DOURNON Geneviève, 1993, Le Salon de Musique du Musée de l'Homme. Édition revue et augmentée. Paris : Muséum National d'Histoire naturelle.

DOURNON Geneviève, 1996, Guide pour la collecte des musiques et instruments traditionnels. Édition revue et augmentée. Paris : UNESCO.

GROGNET Fabrice, 1997-98, D'un Trocadéro à l'autre, histoire de métamorphoses. Mémoire de DEA. Paris : Muséum National d'Histoire Naturelle.

HAGGERTY Michael, 1984, « Georges Henri Rivière : “Un mariage d'Amour”" . Jazz Magazine 325 : 35.

RAULT Lucie, 2000, Instruments de musique du monde. Paris : La Martinière.

ROUGET Gilbert, 1973, « Le département d'ethnomusicologie du Musée de l'Homme ». Revue de Musicologie LIX/1 : 25-33.

SACHS Curt, 1938, Les instruments de musique de Madagascar. Paris : Institut d'Ethnologie.

SHAEFFNER André, 1994 [1936], Origine des instruments de musique. Édition revue et augmentée. Paris : EHESS.

THÉVENIN René, 1935, « Une visite au Musée d'Ethnographie du Trocadéro où l'on trouve les plus riches collections du monde ». Revue Sciences et Voyages (Paris), 21 mars 1935.

\section{NOTES}

1. Les instruments de musique de la collection qui ne portent aucun numéro d'inventaire ou pour lesquels un numéro de substitution a été attribué, faute de pouvoir les rattacher à leur collection 
d'origine (numéro d'inventaire débutant par « $\mathrm{x}$ » ou « x.org. ») n'ont pas pu être pris en compte pour l'établissement de ce schéma.

2. Merci à Nanette Snoep pour les précieuses pistes documentaires qu'elle a bien voulu m'indiquer concernant l'histoire de ces deux institutions.

3. Pour ce qui a trait à l'histoire du département d'ethnomusicologie du Musée de l'Homme, voir Rouget 1973.

4. Voir l'entretien donné par Georges-Henri Rivière à Michael Haggerty (Haggerty 1984).

\section{RÉSUMÉS}

La collection d'instruments de musique qui sera conservée au musée du quai Branly est constituée d'environ huit mille sept cents instruments de toutes provenances, qui se trouvaient autrefois dans les réserves et les salles publiques du Musée National des Arts d'Afrique et d'Océanie et du Musée de l'Homme. Dans cet article sont évoqués quelques uns des événements de l'histoire de cette collection débutant en 1878, liés au développement de la muséologie et de la recherche ethnographique, et qui ont été déterminants pour l'accroissement et la constitution de cet ensemble aussi bien que pour l'élaboration des projets prévus autour des instruments de musique au musée du quai Branly.

\section{AUTEUR}

\section{MADELEINE LECLAIR}

Madeleine Leclair s'intéresse à l'organologie musicale depuis plus d'une dizaine d'années. De 1992 à 2000 elle a travaillé à l'étude et à la gestion des collections instrumentales au département d'ethnomusicologie du Musée de l'Homme, d'abord auprès de Geneviève Dournon, puis de Lucie Rault. Elle s'occupe, depuis l'automne 2000, des collections ethnomusicologiques au musée du quai Branly. Elle prépare une thèse de doctorat sur un répertoire féminin de musique vocale des Itcha (population d'origine Yoruba) du Bénin. 